\title{
Vernalização em cinco cultivares de morangueiro
}

\author{
Vernalization on five cultivars of strawberry
}

\author{
Marcelo Fontanetti Verdial ${ }^{\text {I }}$ João Tessarioli Neto ${ }^{\text {II }}$ Keigo Minami ${ }^{\text {II }}$ \\ João Alexio Scarpare Filho"II Pedro Jacob Christoffoleti" ${ }^{\text {III }}$ Fabio Vale Scarpare ${ }^{\text {III }}$ \\ Juliano Francisco Barela ${ }^{\text {III }}$ Juan Saavedra del Aguila ${ }^{I I I}$ Ricardo Alfredo Kluge $^{\text {IV }}$
}

\section{RESUMO}

A produção de morango é sazonal, e os melhores preços dos frutos são obtidos fora da estação. Buscando obter frutos fora da estação, o objetivo deste trabalho foi avaliar o efeito da vernalização em mudas de cinco cultivares de morangueiro. As mudas foram produzidas no sistema de vasos suspensos. O delineamento experimental utilizado foi blocos ao acaso em fatorial $5 \times 2 \times 4$, com cinco cultivares ("IACCampinas", "Dover", "Sweet Charlie", "Cartuno" e "Oso Grande”), dois tratamentos (com e sem vernalização das mudas) e quatro tempos, com quatro repetições. Para vernalização, as mudas foram levadas para câmara fria à temperatura de $10 \pm 2^{\circ} \mathrm{C}$ e fotoperíodo de $8 \mathrm{~h}$ de luz dia ${ }^{-1}$ durante 28 dias, e em seguida transplantadas. Foram avaliadas as percentagens de sobrevivência, florescimento, frutificação, emissão de estolhos, a massa e o número de frutos produzidos por planta. A sobrevivência de mudas não foi afetada pela vernalização. Houve efeito significativo no florescimento e na frutificação das plantas vernalizadas. O estolonamento de plantas foi mais precoce nas mudas vernalizadas. Para todas as cultivares e tratamentos, a produção de frutos foi insignificante e comercialmente inviável.

Palavras-chave: Fragaria $x$ ananassa Duch., produção de mudas, requerimento de frio.

\section{ABSTRACT}

The strawberry production is seasonal, and the best fruit prices are obtained during the off season. Seeking to get fruits off season, this research was aimed at evaluating the effect of the seedling vernalization of five strawberry cultivars. The strawberry seedling were produced in suspended pot system. The experimental design was is completely randomized blocks with the factorial design $5 \times 2 \times 4$, with five strawberry cultivars
(IAC-Campinas, Dover, Sweet Charlie, Cartuno and Oso Grande), two treatments (with and without strawberry runners vernalization) and four times. For vernalization, the strawberry seedling was taken to cold chamber at $10 \pm 2^{\circ} \mathrm{C}$, and photoperiod of $8 \mathrm{~h}$ of light day ${ }^{-1}$ for 28 days, after this they were transplanted. The parameters evaluation were survival percentages, flower blooming, fructification, stolons emission, the weight and the number of fruits produced. The strawberry seedling survival was not affected by the vernalization. The results showed statistic differences among this treatments only on flower appearance and fructification of the vernalized plants. The runner emitions were earlier in the vernalized seedlings. For all strawberry cultivars and treatments the production of fruits was very low and the produced fruits were unsuitable for commercialization.

Key words: Fragaria $x$ ananassa Duch., runners production, chilling requeriment.

\section{INTRODUÇÃO}

A variação sazonal dos preços do morango é bastante acentuada. Os melhores preços são obtidos nos meses de abril a junho, quando a oferta do produto é muito pequena, em razão das condições climáticas das regiões produtoras e das características das cultivares exploradas. Este fato justifica a crescente busca, por parte dos pesquisadores, de técnicas que proporcionem aos produtores de morango produções mais precoces (DUARTE FILHO et al., 1999).

A maioria das cultivares de morangueiro atualmente utilizadas no Brasil se comportam como plantas de dia curto, isto é, necessitam que haja

'Programa de Pós-graduação em Fitotecnia, Escola Superior de Agricultura “Luiz de Queiroz” (ESALQ), Universidade de São Paulo (USP), Piracicaba, SP, Brasil. E-mail: cruxi@directnet.com.br.

IIDepartamento de Produção Vegetal, ESALQ/USP, CP 09, 13418-900, Piracicaba, SP, Brasil. In memorian.

IIIDepartamento de Produção Vegetal, ESALQ/USP, CP 09, 13418-900, Piracicaba, SP, Brasil.

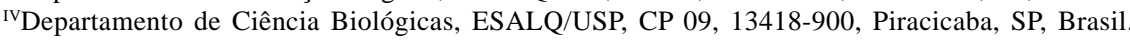


diminuição do fotoperíodo para iniciarem o florescimento e a frutificação. Em condições de temperaturas elevadas e de dias longos, as plantas emitem estolhos que, a cada dois nós (em geral), emitem folhas e enraízam (RONQUE, 1998). Segundo o mesmo autor, os fatores climáticos de maior expressão que afetam a cultura são a temperatura e o fotoperiodismo, sendo que a temperatura exerce maior influência. Fatores como estiagem, chuvas excessivas, alta e baixa umidade e a qualidade e intensidade da luz também exercem influência, mas em menor grau de importância.

Para que ocorra a indução floral, a temperatura e o fotoperíodo devem permanecer favoráveis por um espaço de tempo suficiente, denominado ciclo indutivo. Diversos autores concluíram que o número mínimo de ciclos de dias curtos para induzir o florescimento, nas cultivares octaplóides, está normalmente entre 7 e 14 dias, podendo chegar a 23 dias, conforme citações de GUTTRIDGE(1985).

A vernalização das mudas consiste em fornecer às plantas artificialmente condições de temperatura para que ocorra a diferenciação das gemas em botões florais, na época em que tais condições não ocorrem naturalmente (BALDINI, 1997).

Estudando o efeito da vernalização por 10, 15 e 20 dias, a uma de temperatura de $10^{\circ} \mathrm{C}$ na cultivar de morango “AGF-80”, BRANDÃO FILHO et al. (1989) verificaram que a vernalização teve efeito significativo e que o aumento do tempo de vernalização promoveu a precocidade no aparecimento de botões florais diferenciados, botões visíveis e flores abertas.

Assim, a vernalização de mudas de morangueiro mostra-se como uma possível alternativa para produção de frutos na entressafra, possibilitando maiores ganhos pelos produtores. Visando à obtenção de frutos na entressafra, este trabalho teve como objetivo estudar o efeito da vernalização em mudas de cinco cultivares de morangueiro, avaliando a sua sobrevivência e o potencial de produção na região de Piracicaba, SP.

\section{MATERIAL E MÉTODOS}

O experimento foi conduzido no Departamento de Produção Vegetal, ESALQ/USP, Piracicaba, SP, no período de outubro de 2001 a março de 2003. As cultivares utilizadas foram "IACCampinas”, “Dover”, “Oso Grande”, “Cartuno” e "Sweet Charlie”. As plantas matrizes foram adquiridas da empresa Multiplanta, Andradas, MG. As mudas foram produzidas no sistema de vasos suspensos. Neste sistema, as matrizes foram cultivadas em vasos com a capacidade de 5,0 litros preenchidos com uma mistura de terra tratada com brometo de metila e substrato comercial Geomax, na proporção de 1:1. Os vasos foram mantidos em suportes a $1,50 \mathrm{~m}$ de altura, no espaçamento de $40 \mathrm{~cm}$ entre vasos, dentro de casa de vegetação. Foram utilizadas irrigação por gotejamento e fertirrigação. Os demais tratos culturais, bem como o manejo fitissanitário das plantas matrizes, foi feito conforme a necessidade da cultura.

A coleta das mudas ocorreu no dia 10 de maio de 2002. Estas foram separadas da planta matriz com o auxílio de uma tesoura e colocadas em sacos plásticos de acordo com a cultivar. Após a coleta, foi feita uma padronização de acordo com o tamanho das mesmas em Tipo 1, Tipo 2 e Descarte, de acordo com os parâmetros estabelecidos por ORTIGOZA (1999). Foram utilizadas as mudas do Tipo 2, por representarem a média das mudas produzidas. As mudas selecionadas foram plantadas em bandejas de isopor de 128 células, preenchidas com vermiculita, e levadas para enraizamento em câmara úmida por 15 dias. Após este período, as mudas foram transplantadas para vasos plásticos de $250 \mathrm{ml}$ preenchidos com uma mistura de areia tratada com hipoclorito de sódio a $5 \%$ e vermiculita na proporção de 4:1 (V:V), respectivamente. Para que as mudas pudessem ser testadas na entressafra, estas foram mantidas em casa de vegetação por seis meses em um período de "espera”. Visando ao controle do desenvolvimento das plantas, reduziu-se o fornecimento de nutrientes (20\% do recomendado) e procedeu-se à retirada de flores e estolhos. Durante o período de "espera”, foram feitas três pulverizações com o produto Vertimec para o controle da infestação de ácaros.

As mudas sofreram dois tratamentos (com e sem vernalização). Para a vernalização, as mudas foram colocadas em câmara fria a $10 \pm 2^{\circ} \mathrm{C}$ e fotoperíodo de 8 horas de luz por dia, permanecendo 28 dias nestas condições. Durante este período, a irrigação foi manual, não sendo realizado nenhum tipo de adubação ou tratamento fitossanitário. Para o plantio e condução das mudas no campo, foram feitas aração, gradagem e encanteiramento. Para adubação de plantio, utilizouse o formulado 4-14-8 na quantidade recomendada por PASSOS et al. (1998), conforme análise de solo. O sistema de irrigação utilizado foi por gotejamento através de mangueiras estendidas sobre os canteiros.

No dia 02 de dezembro de 2002, as plantas foram retiradas da câmara fria e, juntamente com as do controle, levadas para as áreas de cultivo em Piracicaba,SP, onde foram transplantadas imediatamente no espaçamento de $30 \mathrm{~cm}$ entre plantas. Três dias após o transplantio, os canteiros foram 
cobertos com plástico preto e foi instalado um túnel baixo para proteção das plantas contra chuva. Procederam-se normalmente aos tratos culturais e ao manejo fitossanitário da cultura.

Foram avaliadas as percentagens de sobrevivência, florescimento, frutificação e emissão de estolhos, além da massa e do número de frutos produzidos por planta. Estas análises foram realizadas após 15, 30, 45 e 60 dias do transplante das mudas para o campo. Os frutos foram colhidos manualmente quando atingiram $70 \%$ de coloração vermelha até completamente maduros, sendo contados e pesados.

Foi utilizado delineamento blocos ao acaso em esquema fatorial $5 \times 2 \times 4$ (cinco cultivares, dois tratamentos com ou sem vernalização e quatro dias de avaliação), com quatro repetições, totalizando 40 tratamentos com 30 mudas cada. Os resultados obtidos foram submetidos à análise de variância (teste F) e as médias dos tratamentos foram comparadas pelo teste de Tukey, ao nível de 5\% de probabilidade de erro. Os dados de percentagem foram transformados em arc. sen. vx/100.

\section{RESULTADOS E DISCUSSÃO}

Pode-se observar que a vernalização das mudas teve efeito sobre a percentagem de sobrevivência de mudas da cultivar "Cartuno" após 60 dias do plantio (Tabela 1). Estes resultados diferem dos obtidos por BALDINI (1997), que verificou uma tendência de queda na sobrevivência de mudas quando estas são vernalizadas. A autora argumenta que, durante a vernalização, as plantas utilizariam suas reservas e, devido a isto, teriam uma menor taxa de sobrevivência quando transplantadas para o campo.

A causa da morte de mudas foi a infecção pelo fungo Colletotrichum fragariae, causador da doença comumente conhecida como “chocolate”. De acordo com TANAKA et al. (2000), esse patógeno de solo tem sua contaminação favorecida por altas temperaturas e umidade elevada, exatamente as condições observadas no período de experimentação.

Comparando as cultivares dentro de cada tratamento, as menores percentagens de sobrevivência foram observadas para a cultivar "Dover" em todas as análises realizadas, sendo que, em alguns casos, os valores foram estatisticamente inferiores aos obtidos pelas demais cultivares. Entretanto, a cultivar "IACCampinas” apresentou as maiores médias para percentagem de sobrevivência de mudas em todas as análises realizadas. Tal fato pode estar relacionado à susceptibilidade ou não de cada cultivar ao ataque do fungo Colletotrichum fragariae.

Considerando esse fungo como o principal agente causal da morte de plantas ao longo do experimento, as diferenças observadas entre as cultivares deve-se ao diferente grau de susceptibilidade ao fungo inerente geneticamente a cada cultivar. Neste caso, a cultivar "IAC-Campinas" demonstrou ser a mais resistente e a cultivar "Dover" a mais susceptível ao fungo.

A percentagem de florescimento de plantas foi diretamente afetada pela vernalização para todas as cultivares, tanto aos 15 quanto aos 30 dias após o transplantio, sendo que as mudas vernalizadas apresentaram médias estatisticamente superiores às das mudas não vernalizadas para todas as cultivares (Tabela 2). A única cultivar que apresentou plantas florescidas no tratamento sem vernalização aos 15 dias foi a "Oso Grande” (2,31\%), sendo que as demais não apresentaram nenhuma planta florescida nesta data. Aos 30 dias após o transplantio, as cultivares "IACCampinas" e “Oso Grande” apresentaram 20,03 e

Tabela 1 - Percentagem de sobrevivência das mudas de diferentes cultivares de morangueiro aos 15, 30, 45 e 60 dias após o transplante em função da vernalização (Vern) ou não (Test) das mudas, Piracicaba/SP, 2003.

\begin{tabular}{|c|c|c|c|c|c|c|c|c|}
\hline & \multicolumn{2}{|c|}{15 dias* } & \multicolumn{2}{|c|}{30 dias* } & \multicolumn{2}{|c|}{45 dias* } & \multicolumn{2}{|c|}{60 dias* } \\
\hline & Vern. & Test. & Vern. & Test. & Vern. & Test. & Vern. & Test. \\
\hline $\begin{array}{l}\text { IAC- } \\
\text { Campinas }\end{array}$ & $100,00 \mathrm{aA}$ & $100,00 \mathrm{aA}$ & 87,16 aA & $90,50 \mathrm{aA}$ & $81,05 \mathrm{aA}$ & $89,39 \mathrm{abA}$ & $72,39 \mathrm{abA}$ & $72,01 \mathrm{abA}$ \\
\hline $\begin{array}{l}\text { Oso } \\
\text { Grande }\end{array}$ & 85,36 abA & 85,99 abA & $75,50 \mathrm{abA}$ & 73,83 aA & 67,17 aA & $64,39 \mathrm{bA}$ & 49,69 bcA & 48,41 bA \\
\hline $\begin{array}{l}\text { Sweet } \\
\text { Charlie }\end{array}$ & $86,74 \mathrm{abA}$ & $93,85 \mathrm{abA}$ & $72,16 \mathrm{abA}$ & 93,83 aA & 76,17 aA & $94,94 \mathrm{aA}$ & $63,25 \mathrm{abA}$ & $94,28 \mathrm{aA}$ \\
\hline Cartuno & 85,99 abA & $80,00 \mathrm{abA}$ & $80,50 \mathrm{abA}$ & $53,83 \mathrm{bA}$ & 83,83 aA & $50,50 \mathrm{cA}$ & 82,82 aA & $43,04 \mathrm{cB}$ \\
\hline Dover & 69,45 bA & 79,08 bA & $50,50 \mathrm{bA}$ & $40,50 \mathrm{bA}$ & 33,83 bA & 39,38 cA & 33,33 сA & $33,33 \mathrm{cA}$ \\
\hline CV (\%) & \multicolumn{2}{|c|}{32,59} & \multicolumn{2}{|c|}{17,40} & \multicolumn{2}{|c|}{16,40} & \multicolumn{2}{|c|}{4,55} \\
\hline
\end{tabular}

*Para cada período avaliado, as médias seguidas de mesma letra minúscula na coluna e maiúscula na linha não diferem entre si pelo teste de Tukey a 5\% de probabilidade de erro. 
Tabela 2 - Percentagem de florescimento das mudas de diferentes cultivares de morangueiro aos 15 e 30 dias após o transplantio em função da vernalização (Vern) ou não (Test), e Percentagem de frutificação das mudas aos 45 e 60 dias após o transplantio, Piracicaba/SP, 2003.

\begin{tabular}{|c|c|c|c|c|}
\hline & \multicolumn{4}{|c|}{$\%$ florescimento } \\
\hline & \multicolumn{2}{|c|}{15 dias* } & \multicolumn{2}{|c|}{30 dias* } \\
\hline & Vern. & Test. & Vern. & Test. \\
\hline IAC-Campinas & $48,29 \mathrm{bA}$ & $0,00 \mathrm{aB}$ & 55,55 aA & $20,03 \mathrm{aB}$ \\
\hline Oso Grande & $59,23 \mathrm{abA}$ & $2,31 \mathrm{aB}$ & 70,75 aA & $11,58 \mathrm{aB}$ \\
\hline Sweet Charlie & 88,70 aA & $0,00 \mathrm{aB}$ & $74,99 \mathrm{aA}$ & $0,00 \mathrm{bB}$ \\
\hline Cartuno & $80,91 \mathrm{aA}$ & $0,00 \mathrm{aB}$ & $75,00 \mathrm{aA}$ & $0,00 \mathrm{bB}$ \\
\hline Dover & $68,60 \mathrm{abA}$ & $0,00 \mathrm{aB}$ & 53,96 aA & $0,00 \mathrm{bB}$ \\
\hline \multirow[t]{4}{*}{ CV (\%) } & & & & \\
\hline & \multicolumn{4}{|c|}{ \% frutificação } \\
\hline & \multicolumn{2}{|c|}{45 dias* } & \multicolumn{2}{|c|}{60 dias* } \\
\hline & Vern. & Test. & Vern. & Test. \\
\hline IAC-Campinas & $23,12 \mathrm{aA}$ & $26,12 \mathrm{aA}$ & 24,15 abA & 29,45 aA \\
\hline Oso Grande & $11,02 \mathrm{abA}$ & 9,65 aA & $8,10 \mathrm{bA}$ & $6,72 \mathrm{aA}$ \\
\hline Sweet Charlie & 3,76 bA & $0,00 \mathrm{bB}$ & $71,58 \mathrm{aA}$ & $0,00 \mathrm{bB}$ \\
\hline Cartuno & $25,29 \mathrm{aA}$ & $0,00 \mathrm{bB}$ & $31,15 \mathrm{abA}$ & $0,00 \mathrm{bB}$ \\
\hline Dover & $9,65 \mathrm{abA}$ & $0,00 \mathrm{bB}$ & $23,80 \mathrm{abA}$ & $0,00 \mathrm{bB}$ \\
\hline CV (\%) & \multicolumn{2}{|c|}{19,50} & \multicolumn{2}{|c|}{25,99} \\
\hline
\end{tabular}

*Para cada período avaliado, as médias seguidas de mesma letra minúscula na coluna e maiúscula na linha não diferem entre si pelo teste de Tukey a 5\% de probabilidade de erro.

$11,58 \%$ de mudas florescidas, respectivamente, mesmo sem vernalização. Entretanto, as médias foram estatisticamente inferiores às das mudas vernalizadas, demonstrando que, mesmo neste caso, a vernalização teve efeito.

O fato de as cultivares "IAC-Campinas" e “Oso Grande” terem florescido mesmo sem vernalização das mudas demonstra que tais cultivares apresentam um comportamento fisiológico diferente das demais cultivares, que, sem vernalização, tiveram a percentagem de florescimento de plantas nulo. GOPPO et al. (1997), estudando o comportamento de diversas cultivares de morangueiro, constataram que a precocidade de produção e a maturação são variáveis conforme a cultivar. Assim, este comportamento é uma característica fisiológica de cada cultivar.

Outro fator a ser considerado seria o estresse nutricional causado pelo condicionamento das mudas. RONQUE (1998) cita que o estresse nutricional por longos períodos pode induzir o florescimento das plantas quando estas são colocadas em condições favoráveis de desenvolvimento novamente. Neste aspecto, as cultivares "IAC- Campinas" e "Oso grande" seriam mais sensíveis ao florescimento causado por estresse nutricional do que as demais cultivares.

A percentagem de frutificação de plantas aos 45 e 60 dias após o transplantio em função da vernalização ou não das mudas e da cultivar encontram-se na tabela 2. As cultivares "IACCampinas” e "Oso Grande” apresentaram percentagens de frutificação estatisticamente iguais em ambos os tratamentos, aos 45 e 60 dias após o transplante. Para as demais cultivares, a vernalização das mudas proporcionou médias estatisticamente superiores quando comparadas com as das mudas que não foram vernalizadas, já que estas não tiveram nenhuma planta com frutos durante todo o período de condução do experimento. Analisando as cultivares dentro de cada tratamento, a maior percentagem de plantas com frutos foi obtida pela cultivar "Sweet Charlie”, no tratamento convernalização, aos 60 dias.

A percentagem de estolonamento de plantas aos 15, 30, 45 e 60 dias após o transplantio em função da cultivar e da vernalização ou não das mudas encontram-se na tabela 3. Aos 15 dias, as plantas das cultivares "IAC Campinas”, “Oso Grande”, “Sweet Chalie” e "Cartuno" apresentaram estolonamento tanto para mudas vernalizadas quanto para a testemunha. Analisando as cultivares dentro de cada tratamento, aos 15 dias, a cultivar "IAC-Campinas" apresentou maiores valores em relação às demais cultivares em ambos os tratamentos, demonstrando maior precocidade na emissão de estolões. Segundo RESENDE et al. (1999), durante o período de verão, 
Tabela 3 - Percentagem de estolonamento das mudas de diferentes cultivares de morangueiro aos 15, 30, 45 e 60 dias após o transplantio em função da vernalização (Vern) ou não (Test) das mudas, Piracicaba/SP, 2003.

\begin{tabular}{|c|c|c|c|c|c|c|c|c|}
\hline & \multicolumn{2}{|c|}{15 dias* } & \multicolumn{2}{|c|}{30 dias* } & \multicolumn{2}{|c|}{45 dias* } & \multicolumn{2}{|c|}{60 dias* } \\
\hline & Vern. & Test. & Vern. & Test. & Vern. & Test. & Vern. & Test. \\
\hline IAC-Campinas & 16,33 aA & $18,07 \mathrm{aA}$ & $50,90 \mathrm{aA}$ & 82,19 aA & $83,14 \mathrm{aA}$ & $100,00 \mathrm{aA}$ & 87,65 aA & $100,00 \mathrm{aA}$ \\
\hline Oso Grande & 7,68 abA & $6,59 \mathrm{abA}$ & 34,27 aA & 74,33 aA & 39,42 bB & $100,00 \mathrm{aA}$ & 93,30 aA & 100,00 aA \\
\hline Sweet Charlie & $2,81 \mathrm{abA}$ & 2,61 abA & $43,00 \mathrm{aA}$ & $59,04 \mathrm{aA}$ & $57,08 \mathrm{abA}$ & $83,34 \mathrm{abA}$ & 95,83 aA & 96,15 aA \\
\hline Cartuno & $0,00 \mathrm{bA}$ & $2,43 \mathrm{abA}$ & 16,66 aA & $34,14 \mathrm{aA}$ & $60,42 \mathrm{abA}$ & $66,07 \mathrm{bA}$ & 96,67 aA & $100,00 \mathrm{aA}$ \\
\hline Dover & $0,00 \mathrm{bA}$ & $0,00 \mathrm{bA}$ & $20,00 \mathrm{aA}$ & 3,02 bA & $30,00 \mathrm{bB}$ & 89,29 aA & $100,00 \mathrm{aA}$ & $100,00 \mathrm{aA}$ \\
\hline CV (\%) & \multicolumn{2}{|c|}{27,44} & \multicolumn{2}{|c|}{26,47} & \multicolumn{2}{|c|}{18,19} & \multicolumn{2}{|c|}{18,97} \\
\hline
\end{tabular}

*Para cada período avaliado, as médias seguidas de mesma letra minúscula na coluna e maiúscula na linha não diferem entre si pelo teste de Tukey a $5 \%$ de probabilidade de erro.

devido principalmente às altas temperaturas, as plantas passam a produzir estolhos, interrompendo a emissão de flores e, conseqüentemente, a produção de frutos.

A cultivar "Oso Grande" foi a que apresentou maior massa de fruto e a cultivar "IACCampinas” o maior número de frutos produzidos por planta nos dois tratamentos utilizados (Tabela 4). As mudas vernalizadas apresentaram maiores médias do que as não vernalizadas, tanto para massa quanto para número de frutos produzidos por planta.

As médias obtidas em relação à massa e ao número de frutos produzidos por planta foram praticamente insignificantes em comparação a dados de produção. Embora a vernalização das mudas tenha propiciado o aumento na percentagem de florescimento e frutificação das plantas, a produção foi drasticamente afetada pelas altas temperaturas ocorrentes no período (os frutos formados apresentaram má formação e tamanho bastante reduzido).

A frutificação é grandemente prejudicada nos trópicos, onde temperaturas mais elevadas fazem com que o morangueiro continue na fase vegetativa, produzindo estolhos indefinidamente, de acordo com RONQUE(1998).
Apesar de ter havido florescimento e frutificação de plantas, o desenvolvimento dos frutos quantitativamente foi comercialmente inviável.

\section{CONCLUSÕES}

A vernalização não afetou a sobrevivência de mudas de nenhuma das cultivares até os 45 dias após o plantio no campo. Entretanto, aos 60 dias após a vernalização, mudas da cultivar "Cartuno" apresentaram maiores percentagens de sobrevivência em relação às demais. A utilização da vernalização foi significativa no florescimento de todas as cultivares e na frutificação das cultivares "Sweet Charlie", "Cartuno" e "Dover". De forma geral, a emissão de estolões foi desfavorecida pela vernalização até os 45 dias após o transplante.

\section{AGRADECIMENTOS E APRESENTAÇÃO}

Ao Conselho Nacional de Desenvolvimento Científico e Tecnológico (CNPq), pela bolsa de produtividade em Pesquisa do quarto, do quinto e do nono autor, e à Fundação

Tabela 4 - Massa e número de frutos por planta em função da cultivar e da vernalização (Vern) ou não (Test) das mudas, Piracicaba/SP, 2003.

\begin{tabular}{|c|c|c|c|c|}
\hline & \multicolumn{2}{|c|}{ Massa (g) } & \multicolumn{2}{|c|}{ Número de frutos } \\
\hline & Vern. & Test. & Vern. & Test. \\
\hline IAC-Campinas & $0,33 \mathrm{cB}$ & $0,71 \mathrm{aA}$ & $3,20 \mathrm{aA}$ & $1,03 \mathrm{aB}$ \\
\hline Oso Grande & $0,86 \mathrm{abA}$ & $0,00 \mathrm{bB}$ & 1,93 abA & $0,00 \mathrm{bB}$ \\
\hline Sweet Charlie & 0,27 bA & $0,00 \mathrm{bB}$ & 1,38 bA & $0,00 \mathrm{bB}$ \\
\hline Cartuno & $0,58 \mathrm{bA}$ & $0,00 \mathrm{bB}$ & 0,97 bcA & $0,00 \mathrm{bB}$ \\
\hline Dover & $1,43 \mathrm{aA}$ & $0,00 \mathrm{bB}$ & 0,58 cA & $0,00 \mathrm{bB}$ \\
\hline CV (\%) & \multicolumn{2}{|c|}{20,91} & \multicolumn{2}{|c|}{13,10} \\
\hline
\end{tabular}

Para cada período avaliado, as médias seguidas de mesma letra minúscula na coluna e maiúscula na linha não diferem entre si pelo teste de Tukey a 5\% de probabilidade de erro.

Ciência Rural, v.37, n.4, jul-ago, 2007. 
de Amparo à Pesquisa de São Paulo (FAPESP), pela bolsa de estudo ao nível de Doutorado do primeiro e do oitavo autor.

Parte da tese de doutorado do primeiro autor, Programa de Pós-graduação em Fitotecnia, Escola Superior de Agricultura “Luiz de Queiroz”, Universidade de São Paulo (USP), Piracicaba, SP, Brasil.

\section{REFERÊNCIAS}

BALDINI, E.M. Vernalização de duas cultivares de morango: produção e análise econômica. 1997. $54 \mathrm{f}$. Dissertação (Mestrado em Fitotecnia) - Escola Superior de Agricultura "Luiz de Queiroz”, Universidade de São Paulo.

BRANDÃO FILHO, J.U.T. et al. Efeito do tempo de vernalização sobre o florescimento do morangueiro. Horticultura Brasileira, v.7, n.45, p.11, 1989.

DUARTE FILHO, J. et al. Aspectos do florescimento e técnicas empregadas objetivando a produção precoce em morangueiros. Informe Agropecuário, v.20, n.189, p.30-35, 1999.

GOPPO, G.A. et al. A cultura do morangueiro. 2.ed Campinas: CATI, 1997. 27p. (CATI. Boletim técnico, 201). GUTTRIDGE, C.G. Fragaria $\mathrm{x}$ ananassa. In: HALVEY, A.H
CRC handbook of flowering. Boca Raton: CRC, 1985. V.3, p.16-33.

ORTIGOZA, L.E.R. Comportamento de diferentes cultivares de morangueiro na produção de mudas de campo. 1999. 43f. Dissertação (Mestrado em Fitotecnia) Escola Superior de Agricultura “Luiz de Queiroz”, Universidade de São Paulo.

PASSOS, F.A. et al. Morango. In: INSTITUTO AGRONÔMICO (Campinas, SP). Instruções agrícolas para o Estado de São Paulo. 6.ed. Campinas: IAC, 1998. p.222-225. (IAC. Boletim, 200).

RESENDE L.M. de A. et al. Panorama da produção e comercialização do morango. Informe Agropecuário, v.20, n.198, p.5-19, 1999.

RONQUE, E.R.V. Cultura do morangueiro: revisão prática Curitiba: EMATER-PR, 1998. 206p.

TANAKA M.A.S. et al. Manejo integrado de pragas e doenças do morangueiro. São Paulo: Secretaria de Agricultura e Abastecimento, 2000. V.5, 61p. (Manual técnico, série especial). 